\title{
Do quantum dots allow one access to pseudogap Kondo physics?
}

\author{
John Hopkinson * , Karyn Le Hur, and Émilie Dupont \\ Département de Physique, Université de Sherbrooke, Sherbrooke, Québec, Canada
}

\begin{abstract}
For the last decade[1], tunable quantum dot systems have allowed the investigation of Kondo physics wherein the quenching of a single spin on an artificial atom affects the conductance. The pseudogap Kondo model (pKm) featuring a density of states $\rho(\epsilon)=\mathrm{C}|\epsilon|^{r}$, introduced by Withoff and Fradkin[2] in 1990 was predicted to exhibit Kondo-like physics above a critical value of the Kondo coupling, $\mathrm{J}_{c}$, which several groups have shown by numerical renormalization group $(\mathrm{RG})$ is finite for $\mathrm{r}<\frac{1}{2}$. Gonzalez-Buxton et al [3] showed that the strong coupling limit of the particle-hole symmetric model leads to a non-trivial $\frac{\pi(1-r)}{2}$ phase shift at low temperatures indicating incomplete screening of the local moment, while away from particle-hole symmetry (p-hs) one generically flows towards a ground state with $\delta \sim \pi$. We examine the implications of this model for quantum dots whose leads are Fermi-liquid-like, yet possess a tunneling density of states (TDOS) which is suppressed at the Fermi energy as a power law.
\end{abstract}

Key words:

pseudogap Kondo model, quantum dots, quantum critical point

Many of the unusual features arising in strongly correlated electron systems have been attributed to the proximity to quantum critical points. In particular, heavy fermion systems are thought to feature a competition between RKKY and Kondo interactions, within the context of a Kondo lattice model, leading to (an increasingly heavy) metallic antiferromagnetic phase which is suppressed as a function of pressure, chemical substitution or magnetic field to produce a cone of nonFermi liquid physics preceeding a (decreasingly heavy) metallic state. Unfortunately, despite decades of intensive research on the Kondo lattice model, the detailed nature of its solution remains a subject of some debate. A simpler model exhibiting a quantum phase transition between a state with local moments and spin-screened state, the pKm, was introduced in Ref. 2 and studied extensively by subsequent groups[3,4,5]. This paper explores the possibility that this model could be experimentally realizable in a quantum dot system.

\footnotetext{
* Corresponding Author: Université de Sherbrooke, QC, CA Phone: (819) 821-8000 ext. 3043 Fax: (819) 821-8046

1 This work was supported by NSERC, FQRNT and CIAR.
}

Resonant level limit We consider the small dot, weak tunneling limit in which the dot is a collection of discrete levels of average spacing $\delta \mathrm{E}$, an order of magnitude smaller than the charging energy, $\mathrm{E}_{c}\left(\mathcal{O}\left(\frac{e^{2}}{2 C}\right)\right)$, of the dot (e: electron charge; C: capacitance of dot-lead system). The energy to add or subtract an electron from the dot can be estimated as $\delta \mathrm{E}_{c}^{ \pm}=\mathrm{E}_{c}\left(\mathrm{Q}_{G}, \mathrm{n}+1\right)$ - $\mathrm{E}_{c}\left(\mathrm{Q}_{G}, \mathrm{n}\right)=\left(\mathrm{n}+\frac{1}{2}-\mathrm{C}_{G} \mathrm{~V}_{G}\right) \frac{e^{2}}{C}$, where $\mathrm{n}$ is the initial(final) number of electrons on the dot for $+(-), \mathrm{V}_{G}$ is an external gate voltage coupled capacitively $\left(\mathrm{C}_{G}\right)$ to the dot. A resonant condition periodic in the number of electrons on the dot corresponds to $\delta \mathrm{E}_{c}^{+(-)}=0$, $\delta \mathrm{E}_{c}^{-(+)}=\frac{e^{2}}{C}$. For temperatures satisfying $\delta \mathrm{E}<\mathrm{T}<\mathrm{E}_{c}$, the physics of the dot is dominated by Coulomb blockade. For temperatures below $\delta \mathrm{E}$, one can write the current through the dot in the single level LandauerBüttiker (elastic scattering) form,

$I=\frac{2 e}{h} \int_{-D}^{D} \frac{4\left(f_{L}(\epsilon)-f_{R}(\epsilon)\right) \Gamma_{L} \Gamma_{R}\left|\frac{\epsilon}{D}\right|^{2 r} d \epsilon}{\left(\epsilon-\epsilon_{d}\right)^{2}+\left(\Gamma_{L}+\Gamma_{R}\right)^{2}\left|\frac{\epsilon}{D}\right|^{2 r}}$.

$f_{L(R)}(\epsilon)$ is the Fermi function of the left(right) lead, 
we have assumed that we are at sufficiently low $\mathrm{T}$ that the intervals $\{ \pm D, \pm \delta E\}$ contribute weakly so we can neglect multiple states and have chosen a powerlaw dot-lead hybridization $\Gamma(\epsilon)=\Gamma_{0}\left|\frac{\epsilon}{D}\right|^{r}$ where $\Gamma_{L}=$ $\Gamma_{R}=\Gamma_{0}=\pi(r+1) t_{k} t_{k^{\prime}} /(2 D)\left(t_{k}\right.$ : dot-lead hopping, $|\epsilon|<D)$. Implicit is the assumption of equivalent leads and dot-lead couplings, and that the effect of electron-electron interactions within the leads is completely captured by a renormalization of the TDOS of the conduction electrons, (in the spirit of the one-dimensional ( $1 \mathrm{D})$ wire RG of Nazarov and Glazman[6]). Using (1) we can calculate the bare conductance $\left(\mathrm{G}_{0}=\left.\frac{d I}{d V}\right|_{V \rightarrow 0}\right)$. Notice that at $\mathrm{T}=0$ the manifestation of Coulomb blockade is an infinitely sharp transmission amplitude at resonance $\left(\epsilon_{d}=0\right)$.

Kondo limit For $\mathrm{T}<\delta E$, directly between two resonant points, with an odd number of spins on the dot, the symmetric condition $\delta \mathrm{E}_{c}^{(+)}=\delta \mathrm{E}_{c}^{(-)}=\frac{e^{2}}{2 C}$ allows one to write $H=\sum_{k \alpha \sigma}\left(\epsilon_{k} c_{k \alpha \sigma}^{\dagger} c_{k \alpha \sigma}+\left(t_{k \alpha} c_{k \alpha \sigma}^{\dagger} d_{\sigma}+\right.\right.$ h.c.) $)+\epsilon_{d} n_{d}+U n_{d \downarrow} n_{d \uparrow}$, where $c_{k \alpha \sigma}$ destroys a conduction electron of momentum $\mathrm{k}$, spin $\sigma$, from lead $\alpha$ and we have defined an effective on-site Hubbard repulsion $\mathrm{U}=-2 \epsilon_{d}$, where $\epsilon_{d}$ is the energy of the highest occupied level on the dot. This model is the pseudogap Anderson model (pAm) studied in Ref. 3 with a reduced régime of validity $\left(\mathrm{D} \rightarrow \mathrm{E}_{c}\right.$ in the energy summation). In the limit $-\epsilon_{d}$ (n odd dot energy), $\mathrm{U}+\epsilon_{d}$ (energy of 1st excitation) $>>\Gamma_{0}, \mathrm{~T}$ the Schrieffer-Wölff transformation leads to[3] $H_{K}=$ $\sum_{k, k^{\prime}}\left(J_{k, k^{\prime}} c_{k \alpha}^{\dagger} \frac{\sigma_{\alpha \beta}}{2} c_{k^{\prime} \beta} S+V_{k, k^{\prime}} c_{k \sigma}^{\dagger} c_{k^{\prime} \sigma}\right)$, where the Kondo coupling, $\mathrm{J}_{k, k^{\prime}}=2\left(\frac{1}{\left|\epsilon_{d}\right|}+\frac{1}{\left|U+\epsilon_{d}\right|}\right) \mathrm{t}_{k} \mathrm{t}_{k^{\prime}}$, and the potential scattering $\mathrm{V}_{k, k^{\prime}}=\frac{1}{2}\left(\frac{1}{\left|\epsilon_{d}\right|}-\frac{1}{\left|U+\epsilon_{d}\right|}\right) \mathrm{t}_{k} \mathrm{t}_{k^{\prime}}$ vanishes at the symmetric point $\mathrm{U}=-2 \epsilon_{d}$.

Following Glazman and Raikh[7], the effects of correlation in the leads ( $\mathrm{r}=0$ for free electrons) is to decrease the effective conductance relative to unitarity at the Kondo limit as $\mathrm{G}=\mathrm{G}_{0} \sin ^{2}(\delta)$, a result one might have anticipated from Kane and Fisher's[8] repulsive interactions in Luttinger liquids (there $\mathrm{G}_{0} \rightarrow \mathrm{gG}_{0}$, g: Luttinger parameter). In the Kondo limit, Ref. 3 found that at p-hs, the pKm flows to a strong coupling fixed point corresponding to the $\mathrm{U}=0$ limit of the pAm provided $\Gamma$ exceeds a critical value which increases monotonically with $\mathrm{r}$ to diverge at $\frac{1}{2}$. The phase shift of this model at the Fermi energy is $\delta=\frac{\pi}{2}(1-r \operatorname{sgn}(\epsilon))[3,4]$. However, this strong coupling fixed point is unstable with respect to the potential scattering term $\mathrm{V}$. At the symmetric point, $\mathrm{V}=0$, one should[5] enter the particle-hole symmetric fixed point below the Kondo scale $\mathrm{T}_{\chi} \sim \delta E\left(\frac{J-J_{c}}{J}\right)^{\frac{1}{r}}$. If $\mathrm{V} \neq 0$, for $0<\mathrm{r}<\mathrm{r}^{*}=0.375$, the initial flow is towards the p-hs fixed point. Below a temperature scale $\mathrm{T}_{\chi^{\prime}}=\left|\frac{V}{\delta E}\right|^{\frac{1}{r}} \mathrm{~T}_{\chi}$ the problem flows to an asymmetric strong coupling fixed point [5] whose phase shift[3] about the Fermi energy is $\pi$. While such a phase shift would be opaque to conductance mea- surements, it is interesting to note that in AhanorovBohm experiments[9] a flux corresponding to a phaseshift of $\pi$ has been apparently observed, and remains unexplained.

In Fig. 1 the effect of p-hs breaking is sketched. While one expects to see evidence of Kondo physics $\left(\mathrm{r}<\mathrm{r}^{*}\right)$ at low temperatures of height comparable to the resonant peak, the width of the peak (in $\mathrm{V}_{G}$ ) will similarly sharpen at $\mathrm{T} \rightarrow 0$ to approach a $\delta$-function at $\mathrm{T}=0$, unlike at $r=0$. While single-walled metallic carbon nanotubes can be created with a sufficiently small power law TDOS, their $1 \mathrm{D}$ nature introduces new complications. The relation of these results with those of 1 D treatments[10] where to our knowledge no critical Kondo coupling has yet been found will be discussed elsewhere[11]. However, a small power-law TDOS (due to interactions between electrons) could be obtained with $2 \mathrm{D}$ leads with a non-negligible resistance[12].

References

[1] For a review see: L. Kouwenhoven and L. Glazman, Physics World, Vol. 14, No. 1, 33 (2001).

[2] D. Withoff et al., Phys. Rev. Lett. 64, 1835 (1990).

[3] C. Gonzalez-Buxton et al., Phys. Rev. B 57, 14254 (1998).

[4] K. Chen et al., J. Phys. Cond. Matt. 7, L491 (1995).

[5] K. Ingersent, Phys. Rev. B 54, 11936 (1996).

[6] Yu. V. Nazarov et al., Phys. Rev. Lett. 91, 126804 (2003).

[7] L. I. Glazman and M. É. Raı̌kh, JETP Lett. 47, 452 (1988); T. K. Ng and P. A. Lee, Phys. Rev. Lett. 61, 1768 (1988).

[8] C. L. Kane et al., Phys. Rev. B 46, 15233 (1992).

[9] Y. Ji et al., Science 290779 (2000).

[10] K. Le Hur, Phys. Rev. B 59, R11637 (1999); P. Fröjdh and H. Johannesson, Phys. Rev. B 53, 3211 (1996); A. Furusaki and N. Nagaosa, Phys. Rev. Lett. 72, 892 (1994); E. H. Kim, cond-mat/0106575

[11] J. Hopkinson et al., cond-mat/0407165

[12] E. Slot et al. cond-mat/0403385 A. Bachtold et al., Phys. Rev. Lett. 87, 166801 (2001); A. N. Cleland et al., ibid. 64 1565 (1990); G. L. Ingold et al. in Single charge tunneling, ed. H. Grabert et al., Plenum Press 1992. 

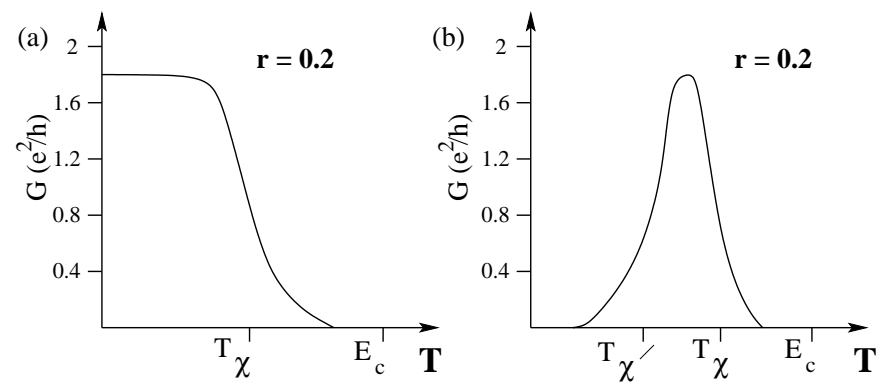

Fig. 1. A schematic sketch for $r=0.2$ of the conductance of the Coulomb blockade valley (a) at p-hs $\mathrm{U}=-2 \epsilon_{d}$; (b) for $\mathrm{V} \neq 0$. 